\title{
Quantitative Thoracic Magnetic Resonance Criteria for the Differentiation of Cysts from Solid Masses in the Anterior Mediastinum
}

\author{
Eui Jin Hwang, $M D^{1}$, MunYoung Paek, $M S^{2}{ }^{2}$, Soon Ho Yoon, $M D^{1}$, Jihang Kim, $M D^{3}$, Ho Yun Lee, MD $^{4}$, \\ Jin Mo Goo, MD ${ }^{1}$, Hyungjin Kim, $M D^{1}$, Heekyung Kim, $M D^{1}$, Jeanne B. Ackman, $M D^{5}$ \\ ${ }^{1}$ Department of Radiology, Seoul National University Hospital, Seoul National University College of Medicine, Seoul, Korea; ${ }^{2}$ Siemens Healthineers, \\ Seoul, Korea; ${ }^{3}$ Department of Radiology, Seoul National University Bundang Hospital, Seongnam, Korea; ${ }^{4}$ Department of Radiology and Center for \\ Imaging Science, Samsung Medical Center, Sungkyunkwan University School of Medicine, Seoul, Korea; ${ }^{5}$ Department of Radiology, Massachusetts \\ General Hospital, Harvard Medical School, Boston, MA, USA
}

Objective: To evaluate quantitative magnetic resonance imaging (MRI) parameters for differentiation of cysts from and solid masses in the anterior mediastinum.

Materials and Methods: The development dataset included 18 patients from two institutions with pathologically-proven cysts $(n=6)$ and solid masses $(n=12)$ in the anterior mediastinum. We measured the maximum diameter, normalized T1 and T2 signal intensity (nT1 and nT2), normalized apparent diffusion coefficient (nADC), and relative enhancement ratio (RER) of each lesion. RERs were obtained by non-rigid registration and subtraction of precontrast and postcontrast T1-weighted images. Differentiation criteria between cysts and solid masses were identified based on receiver operating characteristics analysis. For validation, two separate datasets were utilized: 15 patients with 8 cysts and 7 solid masses from another institution (validation dataset 1); and 11 patients with clinically diagnosed cysts stable for more than two years (validation dataset 2). Sensitivity and specificity were calculated from the validation datasets.

Results: nT2, nADC, and RER significantly differed between cysts and solid masses ( $p=0.032,0.013$, and $<0.001$, respectively). The following criteria differentiated cysts from solid masses: RER $<26.1 \%$; nADC $>0.63 ; \mathrm{nT} 2>0.39$. In validation dataset 1 , the sensitivity of the RER, nADC, and nT2 criteria was $87.5 \%, 100 \%$, and $75.0 \%$, and the specificity was $100 \%, 40.0 \%$, and $57.4 \%$, respectively. In validation dataset 2 , the sensitivity of the RER, nADC, and nT2 criteria was $90.9 \%, 90.9 \%$, and $72.7 \%$, respectively.

Conclusion: Quantitative MRI criteria using nT2, nADC, and particularly RER can assist differentiation of cysts from solid masses in the anterior mediastinum.

Keywords: Anterior mediastinum; Prevascular mass; MRI; Thymoma; Thymic cyst

\section{INTRODUCTION}

With the increased utilization of computed tomography

Received 0ctober 8, 2018; accepted after revision January 12, 2019.

Corresponding author: Soon Ho Yoon, MD, Department of Radiology, Seoul National University Hospital, 101 Daehak-ro, Jongno-gu, Seoul 03080, Korea.

- Tel: (822) 2072-2373 - Fax: (822) 743-6386

- E-mail: yshoka@gmail.com

This is an Open Access article distributed under the terms of the Creative Commons Attribution Non-Commercial License (https://creativecommons.org/licenses/by-nc/4.0) which permits unrestricted non-commercial use, distribution, and reproduction in any medium, provided the original work is properly cited.
(CT), incidental detection of mediastinal masses has become more frequent, with reported prevalence of $0.4-0.9 \%$ (1-3). Anterior mediastinal or prevascular masses may represent various diseases, both benign and malignant (4). The differentiation between cysts and solid masses is important because surgery or tissue acquisition is required for solid masses, while treatment is not usually required for nonneoplastic cysts unless complications occur (5).

Contrast-enhanced CT is the standard modality for evaluating anterior mediastinal lesions $(4,6)$. However, differentiating between cystic and solid masses on CT is sometimes not possible, because cysts can be of high attenuation and mimic solid lesions (7), and because of 
pseudo-enhancement (8), especially in small lesions (5). This limitation is partly responsible for the high nontherapeutic thymectomy rate, which has been reported to be $22-68 \%(5,9,10)$.

The International Thymic Malignancy Interest Group suggested a structured approach for anterior mediastinal lesions. The higher soft tissue contrast and tissue characterization properties of magnetic resonance imaging (MRI) make it a prime modality for distinguishing cystic from solid lesions (11). Indeed, MRI has been reported to be useful for characterizing anterior mediastinal masses (12-15). Nevertheless, previous studies have evaluated the magnetic resonance (MR) features of cysts in a qualitative manner. A qualitative evaluation alone may be open to misinterpretation due to observer inexperience and cardiovascular motion; quantitative measurement of MRI parameters may help overcome such limitations. Therefore, the purpose of our study was to develop quantitative MRI criteria differentiation of cystic from solid masses in the anterior mediastinum.

\section{MATERIALS AND METHODS}

This study was approved by the Institutional Review Boards of all participating institutions and informed consent was waived.

\section{Development Dataset}

To develop differentiation criteria, we collected data from two institutions (Seoul National University Hospital [institution A] and Seoul National University Bundang Hospital [institution B]) with the following criteria: 1) MRIs for the evaluation of an anterior mediastinal lesion taken between June 2008 and January 2018; 2) surgical resection of the lesion; and 3) available pathologic diagnosis. Finally, 18 patients were included in the development dataset (15 from institution A, 3 from institution $B$; 10 males, 8 females; mean age, 52.2 years).

\section{Image Acquisition}

MRIs from institution A were obtained with a 3T scanner (MAGNETOM Skyra, Siemens Healthineers, Erlangen, Germany) using a standardized protocol (Table 1).

MRIs from institution B were obtained with $3 \mathrm{~T}$ and 1.5T scanners (MAGNETOM Skyra; Discovery MR750, GE Healthcare, Milwaukee, WI, USA; and Achieva, Philips Healthcare, Best, the Netherlands) using various protocols. The protocols included at least the following sequences: 1) axial T2-weighted images without fat-suppression, 2) axial T1-weighted images without fat-suppression, 3) precontrast and postcontrast axial T1-weighted images with fat-suppression, and 4) diffusion-weighted images (DWIs) with corresponding apparent diffusion coefficient (ADC) maps.

\section{Image Analyses \\ The following image variables were obtained and analyzed:}

Size: One-dimensional measurements of the lesion were performed on precontrast T1-weighted images in three orthogonal planes. The largest value was recorded as the size of the lesion.

Normalized $\mathrm{T} 1$ and $\mathrm{T} 2$ signal intensity (nT1 and $\mathrm{nT} 2$ ): To assess T1 and T2 signal intensities (SIs) of the lesion, SIs of lesions were measured on representative images with regions of interest (ROIs) covering the entire lesion. Sagittal T1-

\section{Table 1. Standardized MRI Protocol in Institution A}

Scanner: MAGNETOM Skyra (Siemens Healthineers)

Position: Supine

Contrast: Dotarem (gadoterate meglumine; Guerbet); dose, $0.1 \mathrm{mmol} / \mathrm{kg}$ body weight; injection rate, $2 \mathrm{~mL} / \mathrm{sec} ; \mathrm{saline} \mathrm{chasing,} 30 \mathrm{~mL}$ Pulse sequences:

1) Triplanar ECG-gated breathhold HASTE images

2) Axial ECG-gated breathhold T2 turbo spin echo images with double inversion recovery

3) Axial DWI with respiratory triggering using prospective acquisition correction technique, b-values $0,100,400$, and $800 \mathrm{~s} / \mathrm{mm}^{2}$

4) Triplanar precontrast T1-weighted Dixon-VIBE in-phase, out-of-phase, fat-suppressed images with breathhold

5) Coronal DCE with multi-breathhold VIBE technique, including two precontrast sequences and 16 postcontrast phases

6) Triplanar postcontrast T1-weighted Dixon-VIBE in-phase, out-of-phase, fat-suppressed images with breathhold, 5 minutes after intravenous administration of magnetic resonance contrast media

DCE = dynamic contrast enhancement, DWI = diffusion-weighted image, ECG = electrocardiogram, HASTE = half-Fourier-acquired singleshot turbo spin echo, VIBE = volume-interpolated breath-hold examination 
weighted in-phase images and sagittal T2-weighted halfFourier-acquired single-shot turbo spin echo (HASTE) images were preferentially used for evaluation of T1 and T2 SIs, respectively. The $\mathrm{nT} 1$ and $\mathrm{nT2}$ values were defined as fraction values; SI of the lesion divided by SI of cerebrospinal fluid (CSF). For measurement of the SI of CSF, the image with the grossly largest CSF space without flow artifacts in the same sequence and was selected. SIs were measured with ROIs covering as large a CSF space as possible.

Normalized $A D C$ ( $n A D C)$ : A parametric $A D C$ map was obtained from DWIs. The ADCs of lesions were measured on representative images with ROIs covering the entire lesion. Afterwards, nADCs were obtained as a fraction value, $A D C$ of the lesion divided by that of CSF. Images with severe motion artifacts precluding adequate discrimination of the lesion on the $A D C$ map were excluded from the analyses.

Relative enhancement ratio (RER): For the quantitative analysis of contrast enhancement, we measured the RER of the lesion. A work-in-progress software (MRI Arithmetics, Siemens Healthineers) was used for non-rigid registration between axial precontrast and postcontrast T1-weighted fat-suppression images and acquisition of parametric maps of pixel-wise RER values. The equation for the RER is as follows:

$$
\operatorname{RER}(\%)=\frac{S I_{\text {Post }}-S I_{\text {Pre }}}{S I_{\text {Pre }}} \times 100
$$

in which $\mathrm{SI}_{\text {Pre }}$ and $\mathrm{SI}_{\text {Post }}$ indicate SI on precontrast and postcontrast images, respectively. Finally, the RER was measured on the parametric map with ROIs covering the entire lesion.

\section{Validation Dataset}

Two validation datasets were collected. Validation dataset 1 included MRIs of 15 patients (8 males and 7 females; mean age, 55.1 years) from another institution (Samsung Medical Center; institution C), with the following criteria: 1) MRIs for the evaluation of an anterior mediastinal lesion taken between March and November 2017; and 2) available pathologic diagnosis. The images were obtained from one of three scanners (MAGNETOM Skyra; Achieva; and MAGNETOM Avanto, Siemens Healthineers). All data included the following sequences: 1) axial T2-weighted HASTE images; 2) axial T1-weighted images without fatsuppression; 3) axial precontrast and postcontrast T1weighted images with fat-suppression; and 4) DWIs with corresponding $A D C$ maps.

Validation dataset 2 included 11 patients from institution A ( 4 males and 7 females; mean age, 54.3 years) with clinically diagnosed cysts with the following criteria: 1 ) MRIs for the evaluation of an anterior mediastinal mass obtained between December 2014 and December 2015; 2) MRI features of a benign cyst (i.e., lack of internal enhancement, mural nodule, wall thickening, or septation) upon qualitative evaluation by an experienced thoracic radiologist (13 years of experience in interpreting thoracic MRI); and 3) no growth for $>2$ years in follow-up imaging. The acquisition protocol was the same as used for the data in the development dataset from institution A.

\section{Observer Tests}

To evaluate whether our criteria can improve the performance of radiologists' qualitative assessment, we conducted observer tests. Two thoracic radiologists (observer 1 and observer 2, 5 and 2 years of experience after residency, respectively) participated in the tests. In the first session, observers qualitatively evaluated all available images from all pathologically confirmed cases and were asked to determine whether the lesion was a cyst or a solid mass. In the second session, observers re-evaluated images after a 2 month washout period, referring to the developed differentiation criteria and results of quantitative measurements.

\section{Statistical Analyses}

We used SPSS version 23.0 (IBM Corp., Armonk, NY, USA) for statistical analyses. Variables were compared using the Mann-Whitney U test. To identify the differentiation threshold, receiver operating characteristic (ROC) analyses were performed. Correlations between variables were evaluated with Spearman rank correlation analyses. Sensitivities and specificities were calculated regarding cyst as a positive diagnosis. Inter-observer agreements were evaluated with Cohen's kappa coefficient. $P$ values $<0.05$ were considered to indicate statistical significance.

\section{RESULTS}

\section{Comparison between Cysts and Solid Masses in Development Dataset}

Among the development dataset, 6 were cysts, while 12 were solid masses. The pathologic diagnosis of cysts included thymic cysts $(n=3)$, a pericardial cyst, 
Table 2. Comparison of Image Variables between Cysts and Solid Masses in Development Dataset

\begin{tabular}{lccr}
\hline Image Variable & Cysts $(\mathrm{n}=6)$ & Solid Masses $(\mathrm{n}=12)$ & $P$ \\
\hline Size $(\mathrm{cm})$ & $4.4(2.8-6.9)$ & $2.6(1.5-11.8)$ & 0.426 \\
$\mathrm{nT}^{\dagger}$ & $0.59(0.21-0.92)$ & $0.28(0.12-0.58)$ & 0.032 \\
$\mathrm{nT}^{\dagger}$ & $1.90(0.99-5.75)$ & $2.16(8.69-1.17)$ & 0.493 \\
$\mathrm{nADC}^{* \dagger}$ & $1.26(0.52-1.51)$ & $0.33(0.18-0.76)$ & 0.009 \\
RER $\left.^{\dagger} \%\right)$ & $5.3(0.4-24.1)$ & $59.7(28.1-770.7)$ & $<0.001$ \\
\hline
\end{tabular}

All data indicate median values with ranges in parentheses. * Missed in two cases (one cyst and one solid mass), '† Fraction values without units. $\mathrm{nADC}=$ normalized apparent diffusion coefficient, $\mathrm{nT} 1=$ normalized $\mathrm{T} 1$ signal intensity, $\mathrm{nT} 2=$ normalized $\mathrm{T} 2$ signal intensity, $\mathrm{RER}=$ relative enhancement ratio a bronchogenic cyst, and a non-specific cyst ( $\mathrm{n}=1$, respectively). Chronic inflammation accompanied two thymic cysts, while none of the cysts showed internal hemorrhage. For solid masses, pathologic diagnoses were thymomas $(n=5)$, thymic carcinomas $(n=3)$, thymic neuroendocrine tumors $(n=2)$, cavernous hemangioma, and Rosai-Dorfman disease ( $n=1$, respectively).

Cysts had significantly higher nT2 (0.59 vs. $0.28 ; p=$ $0.032)$ and $\operatorname{nADC}(1.26$ vs. $0.33 ; p=0.009)$, and lower RER (5.3\% vs. $59.7 \% ; p<0.001)$ than solid masses (Table 2 , Figs. 1-3).

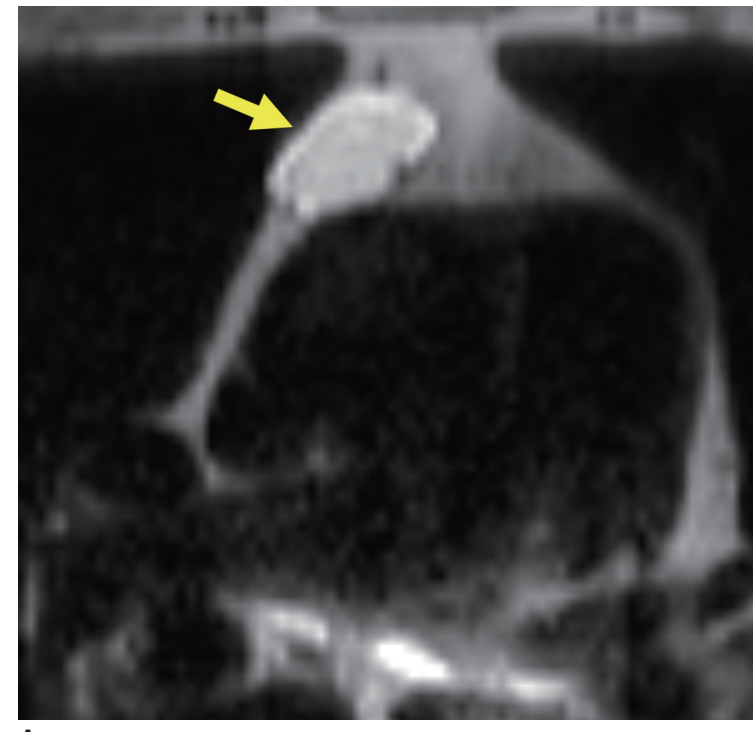

A

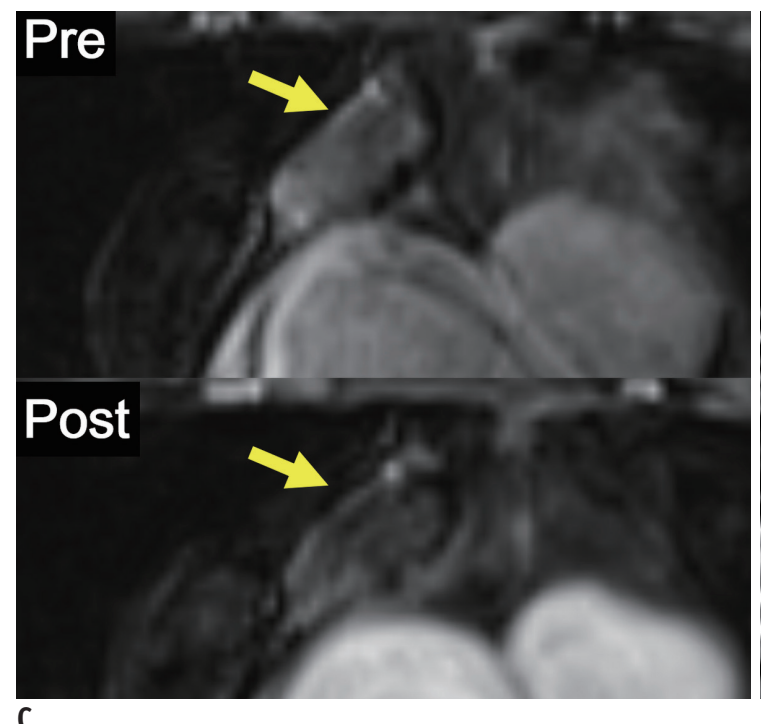

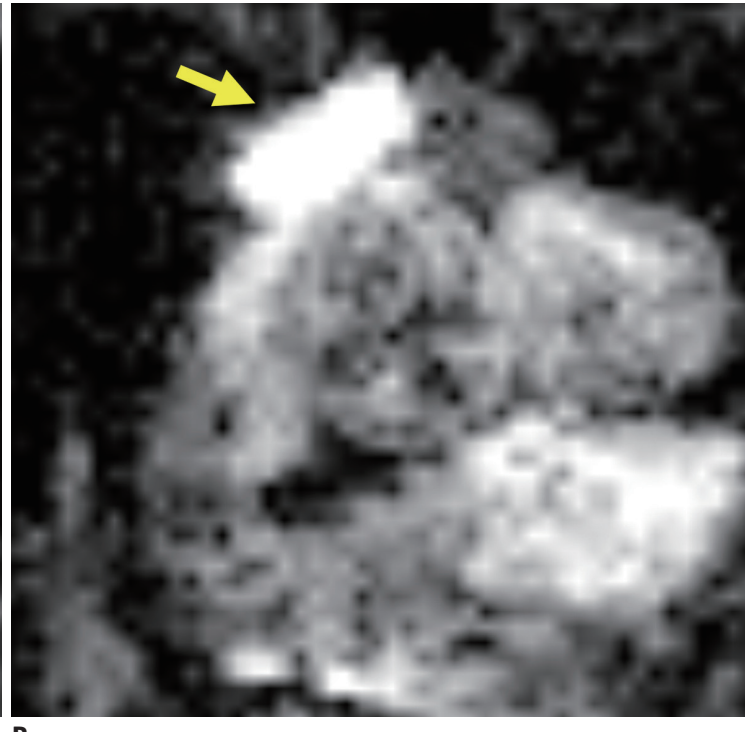

B

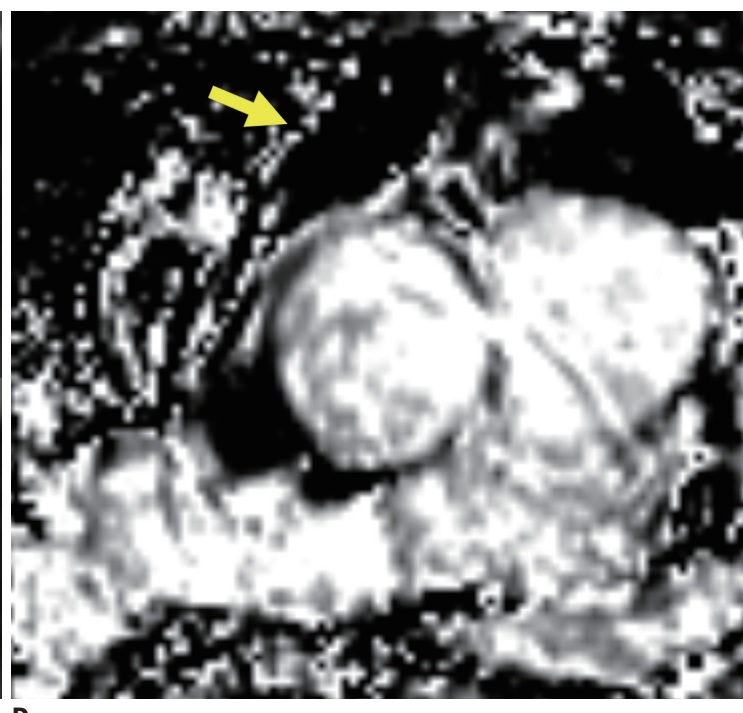

Fig. 1. Representative case from development dataset (cyst).

Axial T2-weighted HASTE image (A), ADC map (B), pre- and post-contrast fat-suppressed T1-weighted images (C), and RER map (D) from 70-year-old male patient revealed $3.1 \mathrm{~cm}$ mass in anterior mediastinum (arrows). nT2, nADC, and RER values were 0.92, 1.50, and 5.3\%, respectively. Pathologic diagnosis after surgical resection was thymic cyst. $A D C=$ apparent diffusion coefficient, HASTE $=$ half-Fourier-acquired single-shot turbo spin echo, $\mathrm{nADC}=$ normalized $\mathrm{ADC}, \mathrm{nT2}=$ normalized $\mathrm{T} 2$ signal intensity, $\mathrm{RER}=$ relative enhancement ratio 

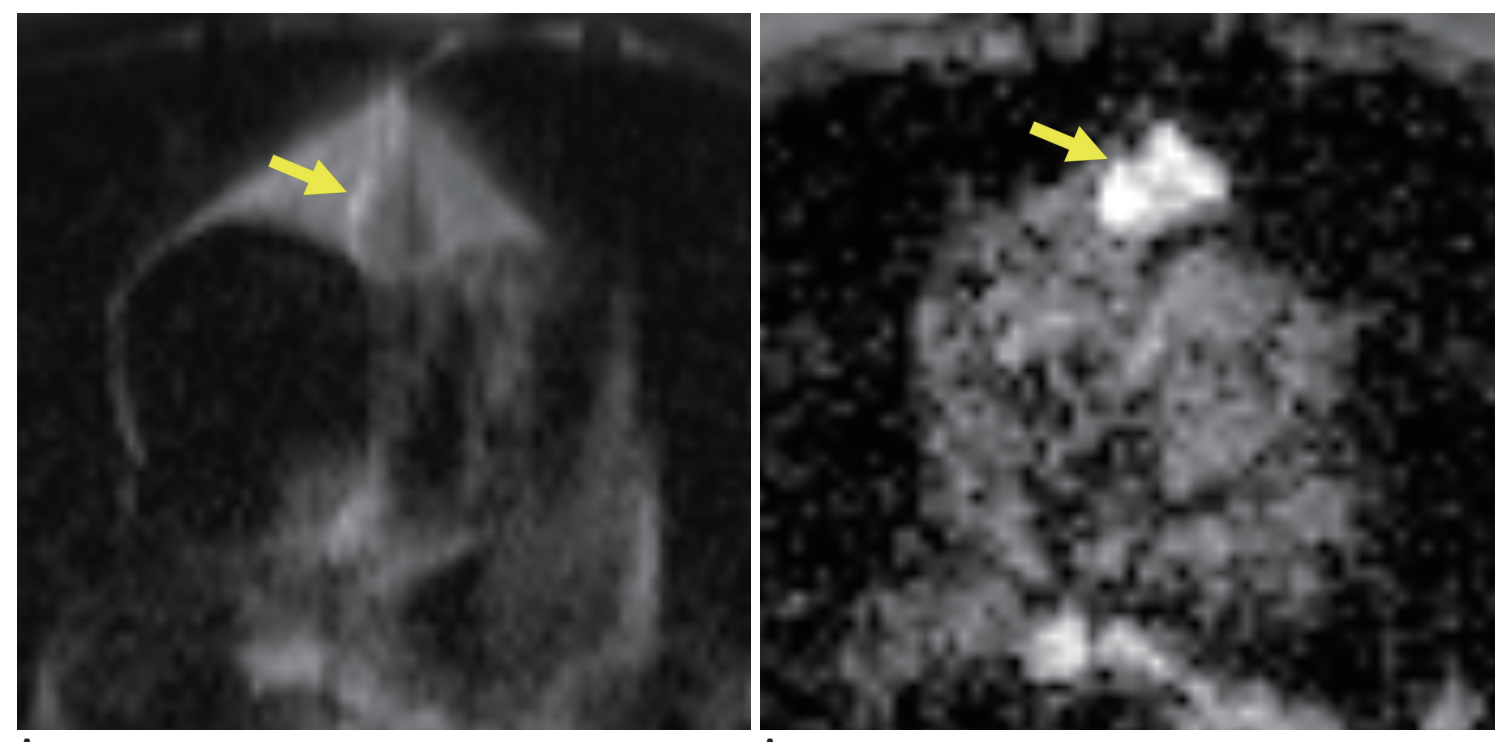

A

A

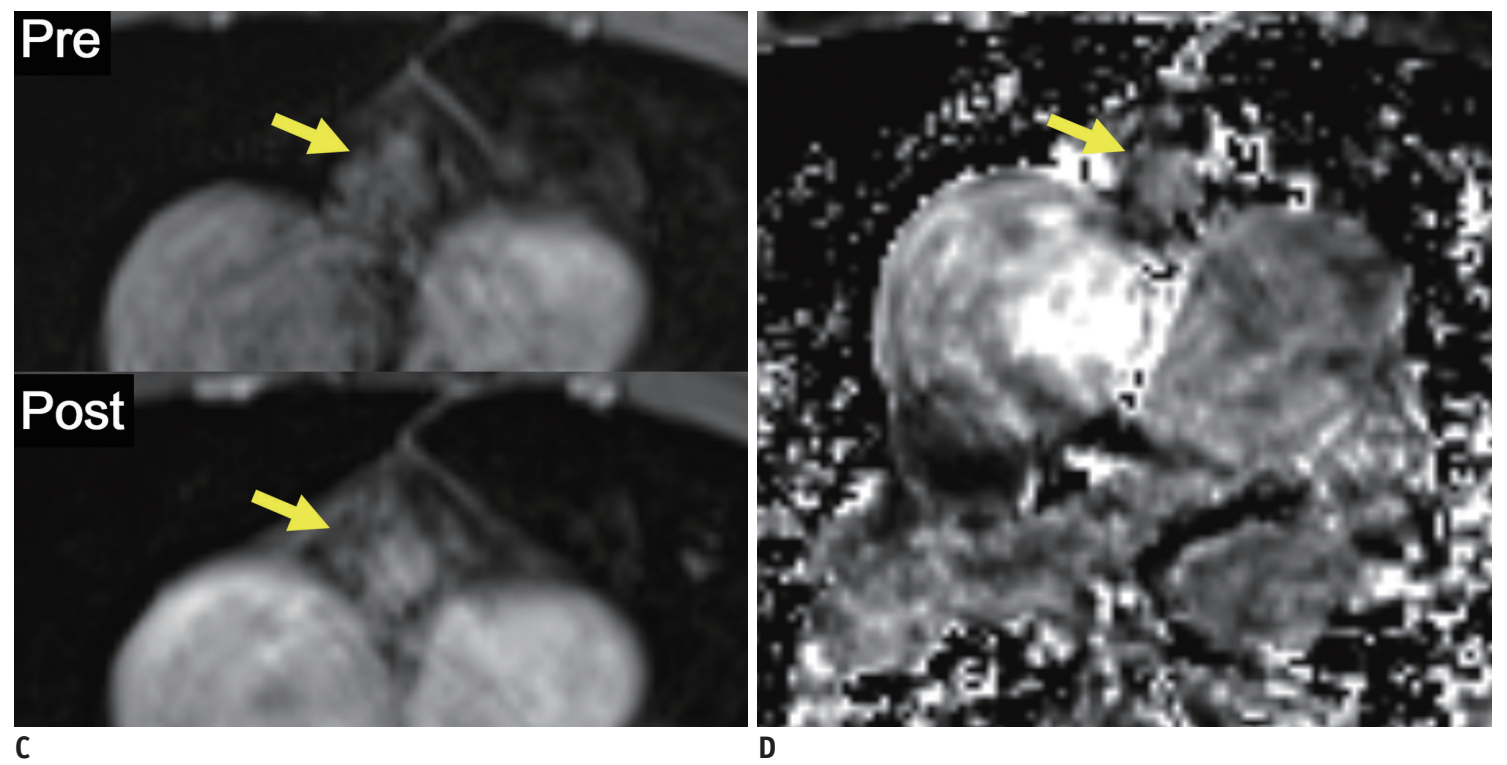

Fig. 2. Representative case from development dataset (solid mass).

Axial T2-weighted HASTE image (A), ADC map (B), pre- and post-contrast fat-suppressed T1-weighted images (C), and RER map (D) from 65-year-old male patient revealed $1.6 \mathrm{~cm}$ mass in anterior mediastinum (arrows). nT2, nADC, and RER values were 0.32, 0.76, and 59.6\%, respectively. Pathologic diagnosis after surgical resection was thymoma, type B2.

\section{Development of Differentiation Criteria}

Table 3 and Figure 3 show the results of the ROC analyses. nT2, nADC, and RER were significant variables for differentiation. Three criteria for differentiating cysts from solid masses were developed: 1) nT2 >0.39; 2) nADC > 0.63; and 3) RER < 26.1\%.

\section{Validation of the Differentiation Criteria}

Among validation dataset 1,8 were cysts and 7 were solid masses. Table 4 shows the results of the comparison between cysts and solid masses in validation dataset 1 . The
RER, ADC, and nT2 criteria showed sensitivities of $87.5 \%$, $100 \%$, and $75.0 \%$, and specificities of $100 \%, 40.0 \%$, and $57.4 \%$, respectively (Table 5 ).

In validation dataset 2 , the median follow-up duration was 34 months (range, 25-54 months). Applying the developed criteria, sensitivities of the RER, ADC, and nT2 criteria were $90.9 \%, 90.9 \%$, and $72.7 \%$, respectively (Table 5 ).

In correlation analyses between image variables, there was a negative correlation between RER and $\mathrm{nT} 2$, with marginal statistical significance $(\rho=-0.490 ; p=0.075)$. 


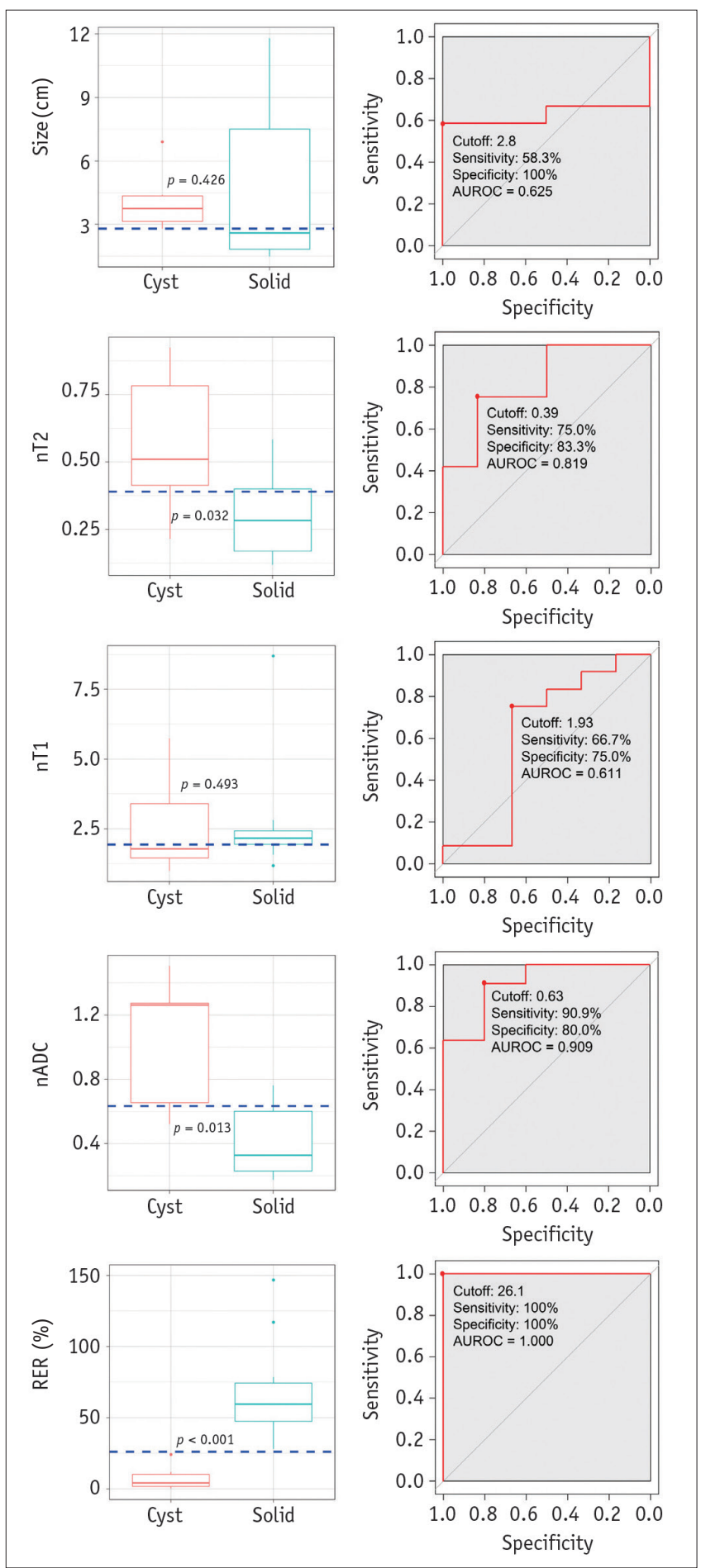

Fig. 3. Boxplots and ROC curves for differentiation between cysts and solid masses in development dataset. Boxplots in left column show results of comparison of image variables between cysts and solid masses. nT2, nADC, and RER differed significantly between cysts and solid masses. Horizontal dashed lines indicate cutoff values developed in ROC analyses. ROC curves in right column show results of ROC analyses for differentiation between cysts and solid masses. Although nT2, and nADC appeared to be significant variables, RER showed perfect separation between cysts and solid masses. nT2, nT1, and nADC are fraction values without units. $A U R O C=$ area under receiver operating characteristic curve, $\mathrm{ROC}=$ receiver operating characteristic
Table 3. Results of ROC Analyses to Define Differentiation Criteria between Cysts and Solid Masses in Development Dataset

\begin{tabular}{lclll}
\hline \multicolumn{1}{c}{$\begin{array}{c}\text { Image } \\
\text { Variables }\end{array}$} & $\begin{array}{c}\text { AUROC } \\
(95 \% \mathrm{CI})\end{array}$ & $\begin{array}{c}\text { Cutoff } \\
\text { Value }\end{array}$ & $P^{*}$ & Youden's J \\
\hline Size $(\mathrm{cm})$ & $0.625(0.360-0.890)$ & 2.8 & 0.399 & 0.583 \\
$\mathrm{nT}^{\ddagger}$ & $0.818(0.592-1.000)$ & 0.39 & 0.047 & 0.618 \\
$\mathrm{nT}^{\ddagger}$ & $0.611(0.282-0.940)$ & 1.93 & 0.454 & 0.417 \\
$\mathrm{nADC}^{\dagger \ddagger}$ & $0.909(0.753-1.000)$ & 0.63 & 0.011 & 0.709 \\
$\mathrm{RER}(\%)$ & $1.000(1.000-1.000)$ & 26.1 & 0.001 & 1.000 \\
\hline
\end{tabular}

*Null hypothesis: true AUROC $=0.5,{ }^{\dagger}$ Missed in two cases (one cyst and one solid mass), 'Fraction values without units. AUROC = area under receiver operating characteristic curve, $\mathrm{CI}=$ confidence interval, $\mathrm{ROC}=$ receiver operating characteristic

Table 4. Comparison of Image Variables between Cysts and Solid Masses in Validation Dataset 1

\begin{tabular}{lccr}
\hline Image Variables & Cysts $(\mathrm{n}=8)$ & Solid Masses $(\mathrm{n}=7)$ & $P$ \\
\hline Size $(\mathrm{cm})$ & $2.4(1.7-6.5)$ & $4.6(1.7-8.2)$ & 0.072 \\
$\mathrm{nT}^{\dagger}$ & $0.64(0.21-1.04)$ & $0.38(0.17-0.77)$ & 0.182 \\
$\mathrm{nT}^{\dagger}$ & $1.63(0.62-3.71)$ & $1.88(0.63-5.25)$ & 0.955 \\
$\mathrm{nADC}^{* \dagger}$ & $1.04(0.99-1.11)$ & $0.70(0.49-1.05)$ & 0.030 \\
RER $(\%)$ & $9.5(2.1-38.0)$ & $97.4(87.1-172.1)$ & $<0.001$ \\
\hline
\end{tabular}

All data indicate median values with ranges in parentheses. * Missed in three cases (one cyst and two solid masses), ${ }^{\dagger}$ Fraction values without units.

\section{Observer Tests}

Results of observer tests are summarized in Table 6 . Both sensitivities and specificities were improved in the second session, in both observers. Inter-observer agreement was also improved when referring to our differentiation criteria.

\section{DISCUSSION}

We quantitatively compared normalized MRI parameters of pathologically confirmed cysts and solid masses in the anterior mediastinum, developed criteria for differentiating between cysts and solid masses, and validated MRI parameter performance on separate datasets.

Bright T2 SIs with high ADCs are of paramount value for diagnosing typical cysts $(7,12,16,17)$. In a recent study (18), an ADC cutoff value of $2.5 \times \cdot 10^{-3} \mathrm{~mm}^{2} / \mathrm{sec}$ showed perfect differentiation between cysts and solid masses in the mediastinum. In our study, although nT2 and nADC differed significantly between cysts and solid masses, substantial overlap was observed, and nADC- and nT2based criteria showed unsatisfactory performance in the validation datasets. This result can be explained by internal 
Table 5. Performances of Differentiation Criteria in Validation Datasets

\begin{tabular}{|c|c|c|c|}
\hline \multirow{2}{*}{ Criteria } & \multicolumn{2}{|c|}{ Validation Dataset 1} & \multirow{2}{*}{$\begin{array}{c}\text { Validation Dataset } 2 \\
\text { Sensitivity (\%) }\end{array}$} \\
\hline & Sensitivity (\%) & Specificity (\%) & \\
\hline RER $<26.1 \%$ & $87.5(47.3-99.7)$ & $100(59.0-100)$ & 90.9 (58.7-99.8) \\
\hline$n A D C>0.63$ & $100(63.1-100)$ & $40.0(5.3-85.3)$ & 90.9 (58.7-99.8) \\
\hline $\mathrm{nT} 2>0.39$ & $75.0(34.9-96.8)$ & $57.4(18.4-90.1)$ & 72.7 (39.0-94.0) \\
\hline
\end{tabular}

Cysts were regarded as positive diagnosis. Data in parentheses indicate $95 \% \mathrm{CI}$.

Table 6. Results of Observer Test

\begin{tabular}{lcc}
\hline \multicolumn{1}{c}{ Dataset } & First Session (Qualitative Reading) & Second Session (Qualitative + Quantitative Reading) \\
\hline Observer 1 & & $100(76.8-100)$ \\
Sensitivity (\%) & $92.9(66.1-99.8)$ & $100(82.4-100)$ \\
Specificity (\%) & $94.7(74.0-99.9)$ & $100(76.8-100)$ \\
Observer 2 & $71.4(41.9-91.6)$ & $100(82.4-100)$ \\
Sensitivity (\%) & $78.9(54.4-93.9)$ & $100(89.4-100)$ \\
Specificity (\%) & & $1.000(1.000-1.000)$ \\
Inter-observer agreement & $75.8(57.7-88.9)$ & \\
\hline Percentage agreement (\%) & $0.504(0.206-0.802)$ & \\
Cohen's kappa
\end{tabular}

Data in the parentheses indicate $95 \%$ CI. Cysts were regarded as positive diagnosis.

hemorrhage or inflammation of cysts, which may alter both the cystic contents and MRI features.

Lack of enhancement is a salient feature for differentiating cysts from solid masses. However, sometimes it is challenging to determine the presence of enhancement, especially in small lesions. In CT images, subtle increased attenuation of cysts after contrast administration or 'pseudo-enhancement' has been well described $(19,20)$. However, in MRI, the degree of enhancement suitable for differentiating cysts from solid masses has rarely been investigated. In our study, we quantitatively evaluated the degree of enhancement in cysts and solid masses after accurate registration of precontrast and postcontrast images. As expected, most cysts showed an RER $<25 \%$, without overlap with RER in solid masses. A cutoff RER of $26.1 \%$ showed excellent performance.

Half (7/14) of pathologically confirmed cysts in our study showed an RER $>10 \%$. This so-called 'MRpseudo-enhancement' might be an important pitfall in differentiation between cysts and solid masses. Motion artifacts, inherent noise, or partial volume averaging with adjacent enhancing structures may have contributed to the MR-pseudo-enhancement (21). In addition, we found a negative correlation between RER and nT2, with marginal significance in pathologically confirmed cysts. In other words, cysts with pseudo-enhancement may show low T2 SI, suggesting that non-water contents due to hemorrhage or inflammation may contribute to pseudo-enhancement.
In our observer tests, misinterpretation of cysts and solid masses occurred in $6-24 \%$ of cases, and interobserver disagreements were noted in $24.2 \%$ of subjective evaluations by radiologists. Impressively, both observers perfectly differentiated cysts and solid masses with the assistance of our differentiation criteria. Although the test was performed in a small population, we believe our quantitative criteria can improve both radiologists' performance and inter-observer agreement.

One aspect worth mentioning is that thymoma may undergo cystic degeneration and show a predominantly cystic appearance (22). There was no cystic thymoma in our population, and our criteria may not work in cystic thymomas. If cystic lesions have an irregular enhancing wall, septation, or mural nodule, the possibility of cystic thymoma should be considered, regardless of quantitative measurement results.

In addition to the intrinsic shortcomings of a retrospective study, our study has several limitations. First, a limited number of pathologically confirmed cases were available. Because patients with definitive cysts on MRI rarely undergo surgery, it was difficult to obtain sufficient pathologically confirmed cases, especially for cysts. Second, as the criteria in our study were developed with data from a small number of scanners with various protocols, it was uncertain whether these criteria would work in data from different scanners or protocols. Although we tried to 
overcome this limitation by normalizing MRI parameters by those of CSF and validating the criteria in independent datasets, further validation with a larger population is required. Finally, for the validation dataset 2, the reference standard was dependent on MRI findings and follow-up imaging, rather than pathologic diagnosis. However, these cases showed MRI features of definitive cysts, in which malignant transformation has been not reported and follow up is not recommended (23).

In conclusion, thoracic MRI with an RER cutoff value of $26.1 \%$ can appropriately differentiate cysts from solid masses of anterior mediastinum, and this criterion can be used to supplement the qualitative assessment.

\section{Conflicts of Interest}

The authors have no potential conflicts of interest to disclose.

\section{ORCID iDs}

Soon Ho Yoon

https://orcid.org/0000-0002-3700-0165

Eui Jin Hwang

https://orcid.org/0000-0002-3697-5542

\section{REFERENCES}

1. Araki T, Nishino M, Gao W, Dupuis J, Washko GR, Hunninghake $G M$, et al. Anterior mediastinal masses in the framingham heart study: prevalence and CT image characteristics. Eur J Radiol Open 2015;2:26-31

2. Henschke CI, Lee IJ, Wu N, Farooqi A, Khan A, Yankelevitz D, et al. CT screening for lung cancer: prevalence and incidence of mediastinal masses. Radiology 2006;239:586-590

3. Yoon SH, Choi SH, Kang CH, Goo JM. Incidental anterior mediastinal nodular lesions on chest $\mathrm{CT}$ in asymptomatic subjects. J Thorac Oncol 2018;13:359-366

4. Shahrzad M, Le TS, Silva M, Bankier AA, Eisenberg RL. Anterior mediastinal masses. AJR Am J Roentgenol 2014;203:W128-W138

5. Ackman JB, Verzosa S, Kovach AE, Louissaint A Jr, Lanuti M, Wright $C D$, et al. High rate of unnecessary thymectomy and its cause. Can computed tomography distinguish thymoma, lymphoma, thymic hyperplasia, and thymic cysts? Eur J Radiol 2015;84:524-533

6. Carter BW, Okumura M, Detterbeck FC, Marom EM. Approaching the patient with an anterior mediastinal mass: a guide for radiologists. J Thorac Oncol 2014;9(9 Suppl 2):S110-S118

7. Tomiyama N, Honda 0 , Tsubamoto M, Inoue A, Sumikawa $H$, Kuriyama K, et al. Anterior mediastinal tumors: diagnostic accuracy of CT and MRI. Eur J Radiol 2009;69:280-288
8. Birnbaum BA, Hindman N, Lee J, Babb JS. Renal cyst pseudoenhancement: influence of multidetector CT reconstruction algorithm and scanner type in phantom model. Radiology 2007;244:767-775

9. Jurado J, Javidfar J, Newmark A, Lavelle M, Bacchetta M, Gorenstein $L$, et al. Minimally invasive thymectomy and open thymectomy: outcome analysis of 263 patients. Ann Thorac Surg 2012;94:974-981; discussion 981-982

10. Kent MS, Wang T, Gangadharan SP, Whyte RI. What is the prevalence of a "nontherapeutic" thymectomy? Ann Thorac Surg 2014;97:276-282; discussion 282

11. Ackman JB, Gaissert HA, Lanuti M, Digumarthy SR, Shepard $J A$, Halpern EF, et al. Impact of nonvascular thoracic MR imaging on the clinical decision making of thoracic surgeons: a 2-year prospective study. Radiology 2016;280:464-474

12. Ackman JB, Wu CC. MRI of the thymus. AJR Am J Roentgenol 2011;197:W15-W20

13. Merine DS, Fishman EK, Zerhouni EA. Computed tomography and magnetic resonance imaging diagnosis of thymic cyst. $J$ Comput Tomogr 1988;12:220-222

14. Inaoka T, Takahashi K, Mineta M, Yamada T, Shuke N, Okizaki $A$, et al. Thymic hyperplasia and thymus gland tumors: differentiation with chemical shift MR imaging. Radiology 2007;243:869-876

15. Zhang W, Zhou Y, Xu XQ, Kong LY, Xu H, Yu TF, et al. A wholetumor histogram analysis of apparent diffusion coefficient maps for differentiating thymic carcinoma from lymphoma. Korean J Radiol 2018;19:358-365

16. Ackman JB. MR imaging of mediastinal masses. Magn Reson Imaging Clin N Am 2015;23:141-164

17. Carter BW, Betancourt SL, Benveniste MF. MR imaging of mediastinal masses. Top Magn Reson Imaging 2017;26:153165

18. Shin KE, Yi CA, Kim TS, Lee HY, Choi YS, Kim HK, et al. Diffusion-weighted MRI for distinguishing non-neoplastic cysts from solid masses in the mediastinum: problemsolving in mediastinal masses of indeterminate internal characteristics on CT. Eur Radiol 2014;24:677-684

19. Bae KT, Heiken JP, Siegel CL, Bennett HF. Renal cysts: is attenuation artifactually increased on contrast-enhanced CT images? Radiology 2000;216:792-796

20. Maki DD, Birnbaum BA, Chakraborty DP, Jacobs JE, Carvalho BM, Herman GT. Renal cyst pseudoenhancement: beamhardening effects on CT numbers. Radiology 1999;213:468472

21. Ho VB, Allen SF, Hood MN, Choyke PL. Renal masses: quantitative assessment of enhancement with dynamic MR imaging. Radiology 2002;224:695-700

22. Romeo V, Esposito A, Maurea S, Camera L, Mainenti PP, Palmieri $\mathrm{G}$, et al. Correlative imaging in a patient with cystic thymoma: CT, MR and PET/CT comparison. Pol J Radiol 2015;80:22-26

23. Daye D, Ackman JB. Characterization of mediastinal masses by MRI: techniques and applications. Appl Radiol 2017;46:10-22 